\title{
EXTENSIONS OF THE FUGLEDE-PUTNAM-TYPE THEOREMS TO SUBNORMAL OPERATORS
}

\section{TAKAYUKI FURUTA}

\begin{abstract}
At first we investigate the similarity between the KleineckeShirokov theorem for subnormal operators and the Fuglede-Putnam theorem and also we show an asymptotic version of this similarity. These results generalize results of Ackermans, van Eijndhoven and Martens. Also we show two theorems on degree of approximation on subnormal derivation ranges. These results generalize results of Stampfli on degree of approximation on normal derivation ranges. The purpose of this paper is to show that the Fuglede-Putnam-type theorem on normal operators can certainly be generalized to subnormal operators.
\end{abstract}

\section{Introduction}

An operator means a bounded linear operator on a complex Hilbert space. Let $B(H)$ denote the set of all bounded linear operators on a complex Hilbert space $H$. An operator $T$ is called subnormal if $T$ has a normal extension.

Following [1], we introduce the following two definitions.

DEFINITION 1. $\left[C_{j}, B\right]_{*}=C_{1} B-B C_{2}$ for $C_{1}, C_{2}, B \in B(H)$.

DEFINITION 2. A topology $\tau$ on $B(H)$ is called an algebra

Received 21 September 1984.

Copyright Clearance Centre, Inc. Serial-fee code: 0004-9727/85 $\$$ A2. $00+0.00$. 
topology, if

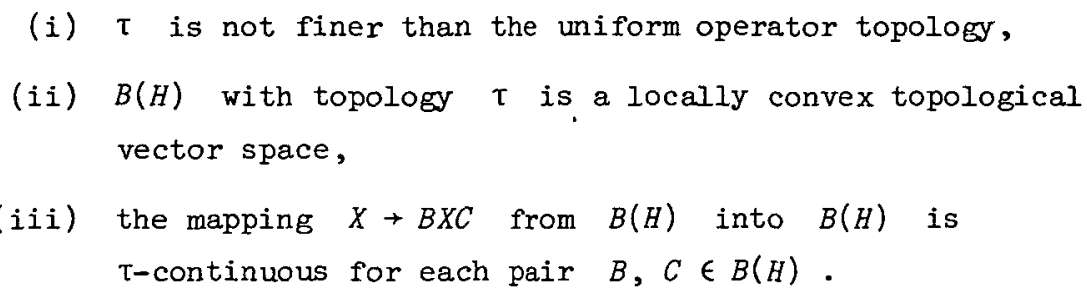

Recently Ackermans, van Eijndhoven and Martens in [1] investigated the similarity between the Kleinecke-Shirokov theorem for normal operators and the Fuglede theorem and also they have shown an asymptotic version of this similarity as follows.

THEOREM A [1]. Let $N_{1}, N_{2} \in B(H)$ be normal. If $\left[N_{j},\left[N_{j}, B\right]_{*}\right]_{*}=0$ then $\left[N_{j}, B\right]_{*}=0$.

THEOREM B [1]. Let $N_{1}, N_{2} \in B(H)$ be normal and let $\tau$ be an algebra topology on $B(H)$. Let $W$ be a t-open neighbourhood of 0 and let $K>0$. Then there exists a t-open neighbourhood $V$ of 0 such that $\left[N_{j}, B\right]_{*} \in W$ for all $B \in B(H)$ with $\|B\| \leq k$ and $\left[N_{j},\left[N_{j}, B\right]_{*}\right]_{*} \in V$.

In [11], Stampfli has estimated the degree of approximation on normal derivation ranges as follows.

THEOREM $C$ [11]. Let $A$ be a normal operator in $B(H)$ with $\sigma(A) \subset \Gamma$ where $\Gamma$ is a rectifiable curve and length $\Gamma=2$. Let $A X-X A=W$. Then there exists a $Y \in B(H)$ and a natural number $n$ such that

$$
\|Y\| \leq n\|X\|
$$

and

$$
\left\|\left(A^{*} Y-Y A^{*}\right)-W\right\| \leq 3 n^{-1} Z\|X\|
$$

THEOREM D [10]. Let $A$ be a normal operator with $\sigma(A) \subset \Gamma$ where $\Gamma$ is a rectifiable curve. Let $\|X\| \leq 1$. If $\|A X-X A\|<\delta$, then $\left\|A^{*} X-X A^{*}\right\|<C \delta^{\frac{1}{2}}$ where $C$ is a universal constant.

Our Theorem 1 shows the similarity between the Kleinecke-Shirokov 
theorem [9], [10], for subnormal operators and the Fuglede theorem [8] and Theorem 1 is an extension of Theorem A. Theorem 2 shows an asymptotic version of this similarity and Theorem 2 is also an extension of Theorem $B$. Theorem 3 and Theorem 4 are extensions of Theorem $C$ and Theorem $D$ respectively. Combining the idea in [3], [4], [5] and [6] for subnormal operators with the results and techniques in Theorem A, Theorem B, Theorem $C$ and Theorem $D$, we show our results. Our main purpose is to show that Fuglede-Putnam type theorems for normal operators such as Theorem A, Theorem B, Theorem $C$ and Theorem D can certainly be generalized to subnormal operators.

\section{Statement of theorems}

First we show Theorem $I$ and Theorem 2. Theorem 1 is an extension of Theorem $\mathrm{A}$ and Theorem 2 is an extension of Theorem B.

THEOREM 1. Let $A_{1}, A_{2}^{*} \in B(H)$ be subnormal. If $\left[A_{j},\left[A_{j}, B\right]_{*}\right]_{*}=0$, then $\left[A_{j}, B\right]_{*}=0$.

THEOREM 2. Let $A_{1}, A_{2}^{*} \in B(H)$ be subnormal and let $\mathrm{I}$ be an algebra topology on $B(H)$. Let $W$ be a t-open neighbourhood of 0 and let $k>0$. Then there exists a $\tau$-open neighbourhood $V$ of 0 such that $\left[A_{j}, B\right]_{*} \in W$ for all $B \in B(H)$ with $\|B\| \leq K$ and $\left[A_{j},\left[A_{j}, B\right]_{*}\right]_{*} \in V$.

Next we show Theorem 3 and Theorem 4. Theorem 3 is an extension of Theorem $\mathrm{C}$ and Theorem 4 is an extension of Theorem D.

THEOREM 3. Let $A$ and $B^{*}$ be subnormal operators in $B(H)$ with $\sigma\left(N_{A}\right) \subset \Gamma$ and $\sigma\left(N_{B^{*}}^{*}\right) \subset \Gamma$ where $N_{A}$ and $N_{B^{*}}$ are normal extensions on $H \oplus H$ of $A$ and $B^{*}$ respectively, and $\Gamma$ is a rectifiable curve and length $\Gamma=2$. Let $A X-X B=W$. Then there exists $a \quad Y \in B(H)$ and $a$ natural number $n$ such that

$$
\|Y\| \leq n\|X\|
$$

and

$$
\left\|\left(A^{*} Y-Y B^{*}\right)-W\right\| \leq 3 n^{-1} Z\|X\|
$$


THEOREM 4. Let $A$ and $B^{*}$ be subnormal operators with $\sigma\left(N_{A}\right) \subset \Gamma$ and $\sigma\left(N_{B^{*}}^{*}\right) \subset \Gamma$ where $N_{A}$ and $N_{B^{*}}$ are normal extensions on $H \oplus H$ of $A$ and $B^{*}$ respectively and $\Gamma$ is a rectifiable curve. Let. $\|X\| \leq 1$. If $\|A X-X B\|<\delta$ then $\left\|A^{*} X-X B^{*}\right\|<C \delta^{\frac{1}{2}}$ where $C$ is a universal constant.

REMARK 1. In Theorem 3 and Theorem 4, assume that $A$ and $B^{*}$ are subnormal operators with $\sigma(A) \subset \Gamma$ and $\sigma(B) \subset \Gamma$ and also assume that they have, respectively, minimal normal extensions $N_{A}$ and $N_{B^{*}}$ on $H \oplus H$, instead of the hypotheses $\sigma\left(N_{A}\right) \subset \Gamma$ and $\sigma\left(N_{B^{*}}^{*}\right) \subset \Gamma$. Then we have the same conclusions as Theorem 3 and Theorem 4 respectively, because $\sigma\left(N_{A}\right) \subset \sigma(A) \subset \Gamma$ and $\sigma\left(N_{B^{*}}^{*}\right) \subset \sigma\left(B^{* *}\right)=\sigma(B) \subset \Gamma$ [8, Problem 157, p.102], so that $A$ and $B^{*}$ satisfy the hypotheses in Theorem 3 and Theorem 4 respectively.

\section{Proofs of theorems}

Proof of Theorem 1. A normal extension $N_{A}$ of $A$ on the Hilbert space $H$ is given by

$$
N_{A_{1}}=\left(\begin{array}{ll}
A_{1} & A_{12}^{(1)} \\
0 & A_{22}^{(2)}
\end{array}\right)
$$

acting on the Hilbert space $H \oplus H$ whose restriction to $H \oplus\{0\}$ is $A$, [7], and a normal one $N_{A_{2}^{*}}$ of $A_{2}^{*}$ on $H$ is also given by

$$
N_{A_{2}^{*}}=\left(\begin{array}{ll}
A_{2}^{*} & A_{12}^{(2)} \\
0 & A_{22}^{(2)}
\end{array}\right)
$$

acting on $H \oplus H$. We put $M_{1}=N_{A_{1}}, M_{2}=\left(N_{A_{2}^{*}}\right)^{*}$, and $\tilde{B}=\left(\begin{array}{ll}B & 0 \\ 0 & 0\end{array}\right)$ on $H \oplus H$. Then $M_{1}, M_{2}$ are both normal on $H \oplus H$. We have $\left[M_{j},\left[M_{j}, \tilde{B}\right]_{*}\right]_{*}=0$ by the assumption as follows: 


$$
\begin{aligned}
{\left[M_{j},\left[M_{j}, \tilde{B}\right]_{*}\right]_{*} } & =M_{1}\left(M_{1} \tilde{B}-\tilde{B} M_{2}\right)-\left(M_{1} \tilde{B}-\tilde{B} M_{2}\right) M_{2} \\
& =\left(\begin{array}{cc}
A_{1}\left(A_{1} B-B A_{2}\right)-\left(A_{1} B-B A_{2}\right) A_{2} & 0 \\
0 & 0
\end{array}\right) \\
& =\left(\begin{array}{cc}
{\left[A_{j},\left[A_{j}, B\right]_{*}\right]_{*}} & 0 \\
0 & 0
\end{array}\right) \\
& =0
\end{aligned}
$$

so that

$$
\left[M_{j}, \tilde{B}\right]_{*}=\left(\begin{array}{cc}
{\left[A_{j}, B\right] *} & 0 \\
0 & 0
\end{array}\right)=0
$$

by Theorem A; that is, $\left[A_{j}, B\right]_{*}=0$, so the proof is complete.

Proof of Theorem 2. As stated in the proof of Theorem 1, we have normal extensions $N_{A_{1}}$ and $N_{A_{2}^{*}}$ on $H \oplus H$ and we employ the same notation as in the proof of Theorem 1 . Also we put $M_{1}=N_{A_{1}}$ and $H_{2}=\left(N_{A_{2}^{*}}\right)^{*}$. We define the subset $W$ as follows:

$$
\tilde{W}=\left\{\left(\begin{array}{ll}
Y_{1} & Y_{2} \\
Y_{3} & Y_{4}
\end{array}\right): Y_{k} \text { in } W(k=1,2,3,4)\right\}
$$

in the set of operators on $H \oplus H$. Then $\tilde{W}$ easily turns out to be a T-open neighbourhood of 0 on $H \oplus H$ because $W$ is so on $H$. By Theorem B, there exists a neighbourhood $\tilde{V}$ of 0 on $H \oplus H$ such that $\left[M_{j}, \tilde{B}\right]_{*} \in \tilde{W}$ for all $\tilde{B} \in B(H \oplus H)$ with $\|\tilde{B}\| \leq K$ and $\left[M_{j},\left[M_{j}, \tilde{B}\right]_{\star}\right]_{*} \in \tilde{V}$.

Define $V=\left\{y:\left(\begin{array}{ll}Y & 0 \\ 0 & 0\end{array}\right)\right.$ is in $\left.\tilde{V}\right\}$. Then this set $V$ turns out to be $a$ $\tau$-neighbourhood of 0 on $H$ because $\tilde{V}$ is so on $H \oplus H$. Assume $B$ is an operator on $H$ with $\left[A_{j},\left[A_{j}, B\right]_{*}\right]_{*}=Y$ in $V$ and $\|B\| \leq K$. Put $\tilde{B}=\left(\begin{array}{ll}B & 0 \\ 0 & 0\end{array}\right)$ on $H \oplus H$. Then $\|\tilde{B}\| \leq K$ and in the same way as in the proof of Theorem 1, we have 


$$
\left[M_{j},\left[M_{j}, \tilde{B}\right]_{*}\right]_{*}=\left(\begin{array}{cc}
{\left[A_{j},\left[A_{j}, B\right]_{*}\right]_{*}} & 0 \\
0 & 0
\end{array}\right)
$$

is in $\tilde{V}$. Hence we have

$$
\left[M_{j}, \tilde{B}\right]_{*}=\left(\begin{array}{cc}
{\left[A_{j}, B\right] *} & 0 \\
0 & 0
\end{array}\right)
$$

is in $\tilde{W}$, which implies that $\left[A_{j}, B\right]_{*}$ is in $W$, so the proof is complete.

In order to prove Theorem 3 we first prove Lemma 1 by employing Berberian's trick [2].

LEMMA 1. Let $A$ and $B$ be normal operators in $B(H)$ with $\sigma(A) \subset \Gamma$ and $\sigma(B) \subset \Gamma$ where $\Gamma$ is a rectifiable curve and length $\Gamma=l$. Let $A X-X B=W$. Then there exists a $Y \in B(H)$ and a natural number $n$ such that

$$
\|Y\| \leq n\|X\|
$$

and

$$
\left\|\left(A^{*} Y-Y B^{*}\right)-W\right\| \leq 3 n^{-1} Z\|X\| \text {. }
$$

Proof. Consider $\tilde{A}, \tilde{X}$ and $\tilde{W}$ on $H \oplus H$ as follows :

$$
\tilde{A}=\left(\begin{array}{ll}
A & 0 \\
0 & B
\end{array}\right), \quad \tilde{X}=\left(\begin{array}{ll}
0 & X \\
0 & 0
\end{array}\right) \text { and } \tilde{W}=\left(\begin{array}{ll}
0 & W \\
0 & 0
\end{array}\right) \text {. }
$$

Clearly $\tilde{A}$ is a normal operator on $H \oplus H$ and $\sigma(\tilde{A})=\sigma(A) \cup \sigma(B) \subset \Gamma$ by the hypotheses and

$$
\tilde{A} \tilde{X}-\tilde{X A}=\left(\begin{array}{cc}
0 & A X-X B \\
0 & 0
\end{array}\right)=\left(\begin{array}{ll}
0 & W \\
0 & 0
\end{array}\right)=\tilde{W} .
$$

By Theorem $\mathrm{C}$, there exists a $\tilde{Y} \in B(H \oplus H)$ and a natural number $n$ such that $\|\tilde{Y}\| \leq n\|\tilde{X}\|$ and

$$
\left\|\left(\tilde{A} * \tilde{Y}-\tilde{Y} \tilde{A}^{*}\right)-\tilde{W}\right\| \leq 3 n^{-1} Z\|\tilde{X}\|
$$

and by scrutinising the proof of Theorem $c$, we have $\tilde{Y}=\left[\theta_{i j} \tilde{X}_{i j}\right]$, where [ ] denotes an operator valued matrix and $\left|\theta_{i j}\right|=1$ for 
$i, j=1,2, \ldots, n$ (see the proof of Theorem $C$ ), that is, there exists a $Y \in B(H)$ such that

$$
\tilde{Y}=\left(\begin{array}{ll}
0 & Y \\
0 & 0
\end{array}\right), \quad Y=\left[\theta_{i j} X_{i, j}\right] \text { and }\|Y\| \leq n\|X\| .
$$

(1) implies

$$
\left\|\left(\tilde{A} * \tilde{Y}-\tilde{Y} \tilde{A}^{*}\right)-\tilde{W}\right\|=\left\|\left(\begin{array}{cc}
0 & \left(A^{*} Y-Y B^{*}\right)-W \\
0 & 0
\end{array}\right)\right\| \leq 3 n^{-1} Z\|\tilde{X}\|=3 n^{-1} Z\|X\|,
$$

so the proof is complete.

Proof of Theorem 3. A normal extension $N_{A}$ of $A$ and a normal one $N_{B^{*}}$ of $B^{*}$ are given by

$$
N_{A}=\left(\begin{array}{ll}
A & A_{12} \\
0 & A_{22}
\end{array}\right), \quad N_{B^{*}}=\left(\begin{array}{ll}
B^{*} & B_{12} \\
0 & B_{22}
\end{array}\right) \text {, }
$$

respectively acting on the Hilbert space $H \oplus H$.

Put $\hat{X}=\left(\begin{array}{ll}X & 0 \\ 0 & 0\end{array}\right)$ and $\hat{W}=\left(\begin{array}{ll}W & 0 \\ 0 & 0\end{array}\right)$ on $H \oplus H$, respectively. Then

$$
N_{A} \hat{X}-\hat{X} N_{B}^{*}=\left(\begin{array}{cc}
A X-X B & 0 \\
0 & 0
\end{array}\right)=\left(\begin{array}{ll}
W & 0 \\
0 & 0
\end{array}\right)=\hat{W},
$$

so that by Lemma $I$, there exists a $\hat{Y} \in B(H \oplus H)$ and a natural number such that $\|\hat{Y}\| \leq n\|\hat{X}\|$ and

$$
\left\|\left(N_{A}^{*} \hat{Y}-\hat{Y} N_{B}\right)-\hat{W}\right\| \leq 3 n^{-1} Z\|\hat{X}\|
$$

By the same way as in the proof of Lemma 1 , we have $\hat{Y}=\left[\theta_{i j} \hat{X}_{i j}\right]$, where [ ] denotes an operator valued matrix and $\left|\theta_{i j}\right|=1$ for $i, j=1,2, \ldots, n$, that is, there exists a $y \in B(H)$ such that

$$
\hat{Y}=\left(\begin{array}{ll}
Y & 0 \\
0 & 0
\end{array}\right), \quad Y=\left[\theta_{i j} X_{i j}\right] \text { and }\|Y\| \leq n\|X\| \text {. }
$$

(2) yields 


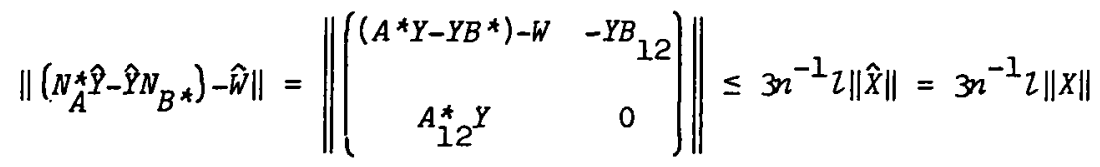

hence

$$
\left\|\left(A^{*} Y-Y B^{*}\right)-W\right\| \leq 3 n^{-1} Z\|X\|,
$$

so the proof is complete.

LEMMA 2. Let $A$ and $B$ be normal operators with $\sigma(A) \subset \Gamma$ and $\sigma(B) \subset \Gamma$ where $\Gamma$ is a rectifiable curve. Let $\|X\| \leq 1$. If $\|A X-X B\|<\delta$ then $\left\|A^{*} X-X B^{*}\right\| \leq C \delta^{\frac{1}{2}}$ where $C$ is a universal constant.

The proof of Lemma 2 is almost the same as that of Lemma 1 using Berberian's trick, so we omit it.

Proof of Theorem 4. We retain the notation of Theorem 3. We have $\|\hat{X}\| \leq 1$ and

$$
\left\|N_{A} \hat{X}-\hat{X} N_{B^{*}}^{*}\right\|=\left\|\left(\begin{array}{cc}
A X-X B & 0 \\
0 & 0
\end{array}\right)\right\|<\delta,
$$

by the hypotheses, then by Lemma 2,

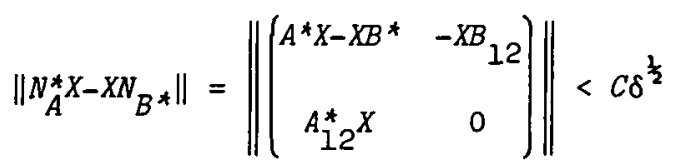

so that

$$
\left\|A^{*} X-X B^{*}\right\|<C \delta^{\frac{1}{2}}
$$

and the proof is complete.

REMARK 2. We remark that Theorem 3 and Theorem 4 do not involve symmetric hypotheses on $A$ and $B$ but rather on $A$ and $B^{*}$. In view of this point, it is natural and reasonable in Lemma $I$ and Lemma 2 to interpret the hypothesis of normality of $A$ and $B$ as that of normality of $A$ and $B^{*}$. 


\section{References}

[1] S.T.M. Ackermans, S.J.L. van Eijndhoven and F.J.L. Martens, "On almost commuting operators", Nederz. Akad. Wetensch. Proc. Ser. A 86 (1983), 385-391.

[2] S.K. Berberian, "Note on a theorem of Fuglede and Putnam", Proc. Amer. Math. Soc. 10 (1959), 175-182.

[3] Takayuki Furuta, "On relaxation of normality in the Fuglede-Putnam theorem", Proc. Amer. Math. Soc. 77 (1979), 324-328.

[4] Takayuki Furuta, "Normality can be relaxed in the asymptotic FugledePutnam theorem", Proc. Amer. Math. Soc. 79 (1980), 593-596.

[5] Takayuki Furuta, "An extension of the Fuglede-Putnam theorem to subnormal operators using a Hilbert-Schmidt norm inequality", Proc. Amer. Math. Soc. 81 (1981), 240-242.

[6] Takayuki Furuta, "A Hilbert-Schmidt norm inequality associated with the Fuglede-Putnam theorem", Bull. Austral. Math. Soc. 25 (1982), 177-185.

[7] Paul R. Halmos, "Shifts on Hilbert space", J. Reine Angew Math. 208 (1961), 102-112.

[8] Paul R. Halmos, A Hilbert space problem book (Van Nostrand, Princeton, New Jersey; Toronto; London; 1967).

[9] D.C. Kleinecke, "On commutators", Proc. Amer. Math. Soc. 8 (1957), $535-536$.

[10] F.V. Shi rokov, "Proof of a conjecture of Kaplansky", Uspekhi Mat. Nauk 11 (1956), 168.

[11] Joseph G. Stampfli, "On self-adjoint derivation ranges", Pacific J. Math. 82 (1979), 257-277.

Department of Mathematics,

Faculty of Science,

Hi rosaki University,

Bunkyo-cho 3,

Hi rosaki 036 Aomori, Japan. 